\title{
ADIABATIC DYNAMICAL CONTROL PHYSICS OF DIELECTRIC MEDIA
}

\author{
R. Grobe \\ Intense Laser Physics Theory Unit, Department of Physics \\ Illinois State University, Normal, IL 61790-4560, USA
}

\begin{abstract}
We summarize recent developments in the area of the coherent propagation of laser pulses through dielectric media. We will show that the principle of dynamical adiabaticity opens new avenues to control the non-perturbative and resonant interaction between two laser pulses and a three-level medium. Examples include the formation of stable wave forms that can travel through optical dielectric media in a loss-free manner with arbitrary pulse shapes, novel possibilities to store optical information in dielectric media in the form of spatially dependent excitations and techniques to exploit this information to control laser pulse envelopes.
\end{abstract}

PACS numbers: $42.65 . \mathrm{Hw}, 42.65 . \mathrm{Re}$

\section{Nonlinear optics and adiabatic dynamical control physics}

In order to describe the spatial and temporal evolution of electromagnetic radiation pulses in dielectric media, the Maxwell equations have to be solved together with the quantum Liouville or Schrödinger equation. The Maxwell equations determine how the electric field (of the laser pulse) evolves as a function of time and space. Its behavior is controlled by the macroscopic polarization of the dielectric medium, which serves as the "source term" in the Maxwell equations. The macroscopic polarization is proportional to the product of the dipole moment and the number density $N$ of the atoms in the medium. The temporal evolution of the polarization is determined by the Liouville equation of the atoms that are driven by the external field. This itself would not be a major complication for the theoretical analysis. If there was just a single differential equation for the polarization, one could use this equation to eliminate the polarization from the Maxwell equations and one would not need to solve for the entire complicated atomic dynamics.

The key problem is that in general there is not just one single differential equation for the polarization but a coupled set of equations that contain several (auxiliary) quantities, such as the inversion or the population of the electronic states that need to be solved simultaneously. A non-perturbative solution of the combined Maxwell and Schrödinger equations is therefore in most cases analytically inaccessible and even numerically it is a demanding computational task. To master this challenge in a computationally more feasible way, it would be helpful to have a more direct relation between the electric field and the polarization. 
As is well known, such a direct relation can be found if the laser field is sufficiently weak and/or its frequency is not near any of the atoms' resonance frequencies. In this case the polarization $P$ can be expanded perturbatively as a standard power series in the electric field amplitude $\mathcal{E}$

$$
P(z, t)=\sum a_{n} \mathcal{E}^{n} \text {. }
$$

In this domain the medium plays a relatively passive role as the atoms get only very weakly excited. This regime is, of course, the realm of nonlinear optics. The optical properties of the medium are modeled by the expansion coefficients $a_{n}$, such as (possibly nonlinear) indices of refraction and higher-order nonlinear susceptibilities. Especially in strongly dissipative atomic systems, the quantum state of the medium can reach quickly its steady state during the duration of the laser pulse and the perturbative expansion above is typically well justified. Using relation (1.1) the polarization as a function of the electric field strength can be inserted back into the Maxwell equations and the problem of solving the atomic equations is elegantly avoided. The resulting set of equations for the electric field can then be solved under various additional approximate assumptions leading, e.g., to nonlinear Schrödinger equations for the field, as shown in many textbooks on linear and nonlinear optics.

The theme of the present work is to describe a different regime in the interaction of laser fields with dielectric media in which another relation between the polarization and the electric field vector can be found. In this regime adiabaticity is the key characteristic to provide this relation. We will describe some recent predictions for this dynamical regime in which the laser field is either relatively strong and/or in resonance with an atomic transition, such that the standard perturbative relation of nonlinear optics between the polarization and the electric field is not valid. We illustrate this regime for the special case of the resonant interaction of a pair of laser pulses with a medium of three-level lambda atoms. This system has recently found some revived interest in the context of electromagnetically-induced transparency $[1,2]$ and also lasing without inversion [3].

For this system it is possible to find a direct relation between the polarization and the electric fields that leads to a fully analytical theory. We assume here that the two laser frequencies are sufficiently different such that each laser pulse excites only one atomic transition. To keep the formalism as transparent as possible and to focus on the essential elements of this approach, we also neglect all dissipative influences. Subsequent work will be devoted to explore how the basic theory needs to be modified to account for relaxation effects.

Under these simplifying assumptions the temporal response of each lambda-atom to the two laser pulses is given by the well-known Schrödinger equation for the state amplitudes $C_{i}$ of the three levels

$$
\begin{aligned}
\mathrm{i} \frac{\partial}{\partial \tau} C_{1}(z, \tau) & =-\frac{1}{2} \Omega_{a}^{*} C_{2}(z, \tau), \\
\mathrm{i} \frac{\partial}{\partial \tau} C_{2}(z, \tau) & =-\frac{1}{2} \Omega_{a} C_{1}(z, \tau)-\frac{1}{2} \Omega_{b} C_{3}(z, \tau), \\
\mathrm{i} \frac{\partial}{\partial \tau} C_{3}(z, \tau) & =-\frac{1}{2} \Omega_{b}^{*} C_{2}(z, \tau)
\end{aligned}
$$


where we have used the rotating wave approximation. With respect to the spatial evolution we have replaced the laboratory time $t$ by a retarded time variable $\tau=t-z / c$. The time and space dependent parameters $\Omega_{a}$ and $\Omega_{b}$ are the Rabi frequencies of the two fields.

One eigenvector of the system of Eqs. (1.2) is the well-known dark or trapped state $[4,5]$ of the form $\left(C_{1}, C_{2}, C_{3}\right) \approx\left(\Omega_{b} / \Omega, 0,-\Omega_{a} / \Omega\right)$, where $\Omega^{2} \equiv \Omega_{a}^{2}+\Omega_{b}^{2}$ is the so-called two-photon Rabi frequency. In the following we will assume that this trapped state condition approximates the response of the atoms to the fields sufficiently well. In practice, this can be achieved via an appropriate sequencing of the turn-on times of the two laser pulses [6]. If we insert this eigenvector back into the right hand side of equations (1.2a) and (1.2c) and solve for the amplitude of the upper state $C_{2}(z, \tau)$, we obtain $C_{2} \approx-2 \mathrm{i} / \Omega_{a}^{*}(\partial / \partial \tau)\left(\Omega_{b} / \Omega\right)=2 \mathrm{i} / \Omega_{b}^{*}(\partial / \partial \tau)\left(\Omega_{a} / \Omega\right)$. This approximation leads then to a direct relation between the effective polarizations (for the reduced wave equation of the Rabi frequencies) and the electric field vector (represented by the Rabi frequencies)

$$
\begin{aligned}
& P_{a}(z, \tau)=\mathrm{i} \mu_{a} C_{1}^{*} C_{2}=-(2 \mu / \Omega) \frac{\partial}{\partial \tau}\left(\Omega_{a} / \Omega\right), \\
& P_{b}(z, \tau)=\mathrm{i} \mu_{b} C_{3}^{*} C_{2}=-(2 \mu / \Omega) \frac{\partial}{\partial \tau}\left(\Omega_{b} / \Omega\right) .
\end{aligned}
$$

The coupling coefficients $\mu_{a, b}$ are related to the number density of atoms $N$ via $\mu_{a, b} \equiv N d_{a, b}^{2} \omega_{a, b} /\left(\varepsilon_{0} \hbar c\right)$, where the dipole moment matrix elements for the transitions 1-2 and 2-3 are denoted by $d_{a}$ and $d_{b}$, respectively.

These relations are in striking contrast to the perturbative relation (1.1) of nonlinear optics. In this case the polarization increases with decreasing electric field strength (which is proportional to $\Omega$ ) and a perturbative expansion would not be valid. Another difference lies in the fact that the size of the polarization depends also on the time derivative of the field. Rapidly changing envelopes in $\Omega_{a}$ and $\Omega_{b}$ induce an enhanced polarization. The polarization for the 1-2 transition depends on both fields $\Omega_{a}$ and $\Omega_{b}$.

Both polarizations vanish completely if the two pulses have identical envelopes and differ only by their amplitudes. In this case the source term Eq. (1.3) for the Maxwell equations is zero and one could conjecture that any two input pulses with matching envelopes evolve in a fully transparent manner with the velocity $c$. However, the corresponding trapped state for matched pulses $(1 / \sqrt{2}, 0,-1 / \sqrt{2})$ does not agree with the state of a medium initially in the ground level $(1,0,0)$. We will see in Sec. 2 that the relations (1.3) force the fields to evolve into "antimatched" pulse shapes to become transparent. This kind of transparency is fully dynamical, that means the polarization is not zero and a significant amount of population gets transferred between the levels as the pulses propagate.

A new exciting research area is based on these relations (1.3). This is an example of what one could call "adiabatic dynamical control physics". As we will argue below, these relations predict the control of nearly arbitrary pulse envelopes and quantum states of the medium. One has to be careful not to confuse the term adiabatic with weak or off-resonant fields. One can show that the inverse of the upper state amplitude $C_{2}$ can serve as a measure for the degree of adiabaticity [7]. 
From the scaling of $C_{2}$ as a function of $\Omega$, it is clear that a stronger Rabi frequency $\Omega$ favors a more adiabatic response. When we use the term adiabatic, from now on we mean "temporally" adiabatic. Its relation to "spatial" adiabaticity in three-level pulse propagation has been discussed in Ref. [8].

In the following sections we will briefly summarize the basic ideas for three interesting phenomena that can be derived analytically from the relations (1.3). These phenomena are exploited in new forms of optical transparency, novel possibilities to control laser pulse shapes and to store optical information in dielectric media in the form of spatially dependent excitations.

\section{Loss-free pulse forms}

The reduced wave equations for the fields in the slowly varying envelope approximation and in one spatial dimension take the well-known form: $(\partial / \partial \tau) \Omega_{a}(z, \tau)=P_{a}(z, \tau)$ and $(\partial / \partial \tau) \Omega_{b}(z, \tau)=P_{b}(z, \tau)$. To keep here the theoretical description analytically feasible, we assume that both atomic transitions have identical oscillator strengths $\mu_{a}=\mu_{b} \equiv \mu$.

Analogous to non-linear optics we can insert the relation between the effective polarization and the electric field into the reduced Maxwell equations, which take the following form [9]:

$$
\begin{aligned}
& \frac{\partial}{\partial \tau} \Omega_{a}=-\frac{2 \mu_{a}}{\Omega} \frac{\partial}{\partial \tau} \frac{\Omega_{a}}{\Omega}, \\
& \frac{\partial}{\partial \tau} \Omega_{b}=-\frac{2 \mu_{b}}{\Omega} \frac{\partial}{\partial \tau} \frac{\Omega_{b}}{\Omega} .
\end{aligned}
$$

Reduced wave equations in general neglect internal reflections of the medium and often also transverse propagation effects. In our case the two equations are nonlinearly coupled via the Rabi frequency $\Omega$. By multiplying the first of the two equations with $\Omega_{a}$ and the second with $\Omega_{b}$ and adding both equations, one can find that the total Rabi frequency is not a function of the position $z, \Omega(z, \tau)=$ $\Omega(z, \tau=0) \equiv \Omega(\tau)$, and it is therefore determined once and forever by the two pulses at the entry surface of the medium at $z=0$. If we choose appropriate initial pulse pairs such that $\Omega(\tau)$ becomes time independent after a characteristic time $T^{*}, \Omega(\tau)=\Omega$ for $\tau>T^{*}$, then it is clear that the wave equations are decoupled and describe the shape invariant propagation of two laser pulses after this time $T^{*}$. In other words, each layer of the medium is passed by the same pulse pair. Measured in the $(z, \tau)$ space-time frame, the velocity of the pulses is $2 \mu / \Omega^{2}$, corresponding to a laboratory velocity $v$ as given by $1 / v=1 / c+2 \mu / \Omega^{2}$. These pulse pairs do not travel with the (vacuum) speed of light $c$, but with velocities that can be several orders of magnitudes smaller than $c$.

Quite remarkably, the two non-linearly coupled partial differential Eqs. (2.1) can be solved fully analytically and require only the inversion of an integral

$$
\Omega_{a, b}(z, \tau)=\frac{\Omega_{a, b}\left[z=0, X^{-1}(X(\tau)-z)\right]}{\Omega\left[z=0, X^{-1}(X(\tau)-z)\right]} \Omega(z=0, \tau),
$$

where $X^{-1}$ denotes the inverse function of the integral $X(\tau) \equiv(1 / 2 \mu) \int^{\tau} \mathrm{d} \tau^{\prime}\left|\Omega\left(\tau^{\prime}\right)\right|^{2}$. 


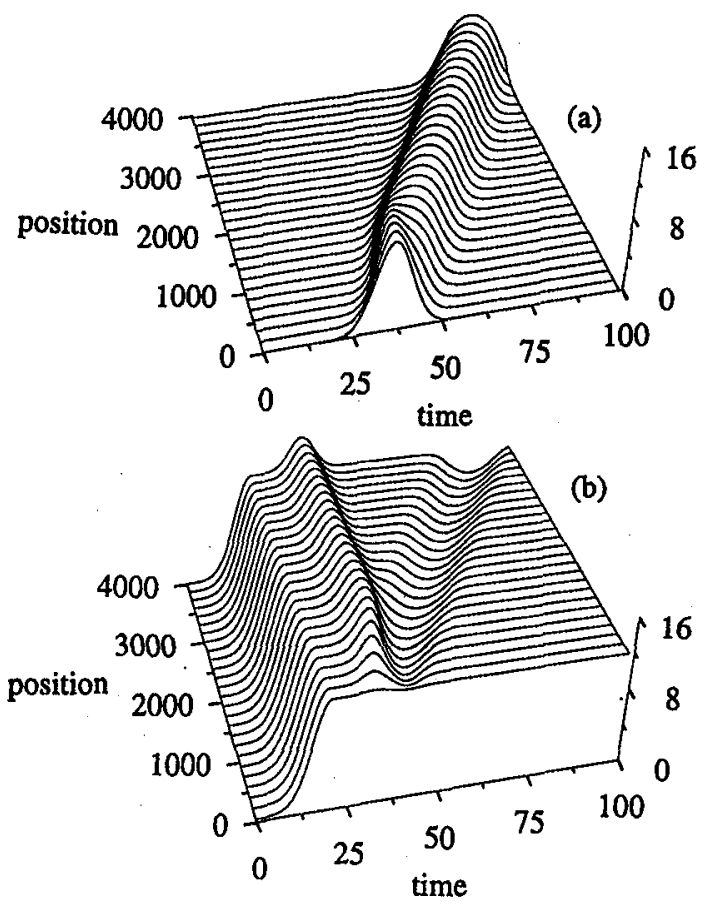

Fig. 1. The formation of loss-free pulses. (a) $\Omega_{a}(z, \tau)$ and (b) $\Omega_{b}(z, \tau)$ as predicted by Eq. (2.2). The parameters are: $\mu=1, \Omega_{a}(z=0, \tau)=A \exp \left[-0.5((\tau-35) / 7)^{2}\right]$, $\Omega_{b}(z=0, \tau)=B \exp \left(-0.5\left[\left(\tau-4 \tau_{\mathrm{p}}\right) / \tau_{\mathrm{p}}\right]^{2}\right)$ for $\tau<4 \tau_{\mathrm{p}}$ and $\Omega_{b}(z=0, \tau)=B$ for $\tau_{\mathrm{p}}<\tau$, $A=10, B=12, \tau_{\mathrm{p}}=5$.

In Fig. 1 we display the evolution of the two pulses as a function of the position $z$ and position $\tau$. The pulses were chosen such that $\Omega$ is constant for $\tau>T^{*}$. Both pulses reshape until after a characteristic propagation distance a shape-invariant and nearly loss-free propagating pulse-pair is formed. The energy of each pulse is conserved during the propagation. The final two "antimatched" pulses are called adiabatons [9]. First experimental evidence of these pulse forms has been reported by the Stanford group of S.E. Harris [10]. We should note here that adiabatons are intrinsically different than solitons, which are exact solutions and take typically specific functional forms $[11,12]$. The difference between adiabatons and electromagnetically induced transparency is discussed in Ref. [13]. In contrast, adiabatons can take arbitrary forms and are characterized by "antimatched" amplitudes such that $\Omega_{a}^{2}(z, \tau)+\Omega_{b}^{2}(z, \tau)$ is independent of time.

\section{Control of the excitation state of three-level media}

There has been a growing interest in studying the optical properties of media that are in a coherent superposition of two states [2, 14-18]. In this section we will address the question whether it is possible to bring the medium into a prescribed excited state with a given spatial dependence. Can one use lasers to 
control the final state of excitation of the medium after interaction with the two pulses? The surprising answer is yes $[19,20]$. As the state of the medium is coupled to the instantaneous amplitudes of the fields via the trapped state relation, we can therefore obtain analytically the temporal and spatial evolution of the medium. The population in the third state $\left|C_{3}\right|^{2}=\rho_{33}$ is given by $\rho_{33}=\left|\Omega_{a}(z, \tau) / \Omega(\tau)\right|^{2}$, whose evolution is known from the solution (2.2). The final state of the medium after both laser pulses have propagated through it takes the following form:

$$
\rho_{33}(z, \tau=T)=\left|\frac{\Omega_{a}\left[z=0, X^{-1}(Z-z)\right]}{\Omega\left[z=0, X^{-1}(Z-z)\right]}\right|^{2} \equiv|G(z)|^{2},
$$

where $T$ is the pulse duration for $\Omega$ and $Z \equiv X(T)$ is the corresponding integral which is proportional to the total energy contained in the input pulses. This result shows that any desired spatially dependent profile $G(z)$ can be obtained with an appropriate choice of two control laser pulses at input. From a mathematical standpoint, however, it is non-trivial to invert Eq. (3.1), that is to solve this equation for $\Omega_{a}$ and $\Omega_{b}$ as a function of a given excitation function $G(z)$.

To illustrate Eq. (3.1) let us present here an illustrative example for which such a mathematical inversion is formally possible by restricting the input pulses shapes to have "antimatched" envelopes such that $\Omega_{a}(z=0, \tau)^{2}+\Omega_{b}(z=0, \tau)^{2} \equiv$ $B^{2}$ is a square pulse for $0<\tau<T_{b}$ and zero otherwise. This, of course, does not necessarily imply that $\Omega_{a}$ and $\Omega_{b}$ are constant. In this case the integral can be solved easily $X(\tau)=\left(B^{2} / 2 \mu\right)\left[\tau-\left(\tau-T_{b}\right) \vartheta\left(\tau-T_{b}\right)\right]$, where $\vartheta$ is the Heaviside unit step function. Its inverse function is $X^{-1}(x)=\left(2 \mu / B^{2}\right) x$ for $0 \leq x<Z$ and it has a singularity at $x=Z=\left(B^{2} / 2 \mu\right) T_{b}$. If we insert this into Eq. (3.1) and solve for $\Omega_{a}(z=0, \tau)$, we obtain the pulse shape of the input pulses as a function of the given excitation profile $G(z)$
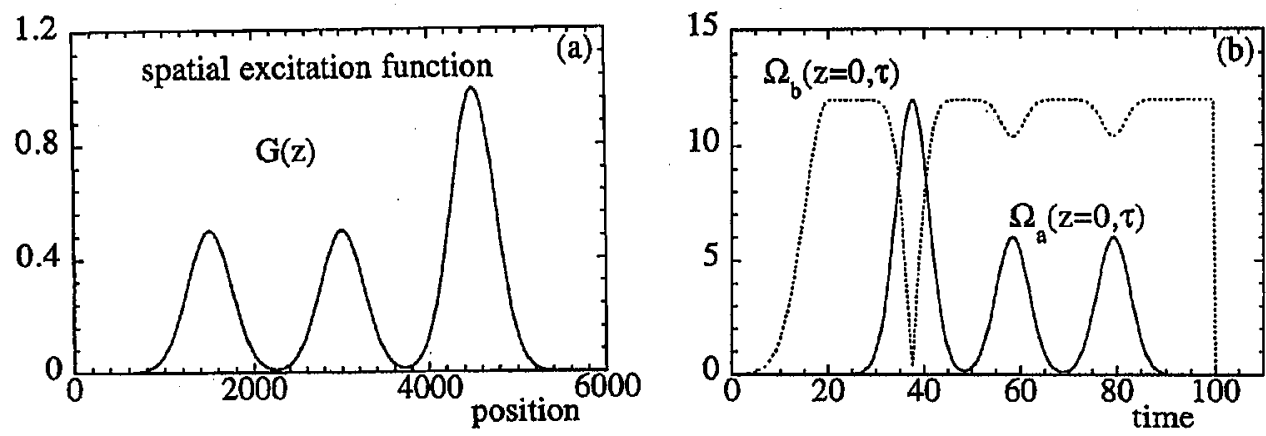

Fig. 2. Generation of spatially dependent excitations. (a) An example for a three-peaked excitation distribution function $G(z), G(z)=0.5\left\{\exp \left[-\left(\left(z-z_{1}\right) / w\right)^{2}\right]+\right.$ $\left.\exp \left[-\left(\left(z-z_{2}\right) / w\right)^{2}\right]\right\}+\exp \left[-\left(\left(z-z_{3}\right) / w\right)^{2}\right]$, where $z_{1}=1500, z_{2}=3000, z_{3}=4500$ and $w=350$. (b) Temporal profiles of the two input laser pulses $\Omega_{a}$ and $\Omega_{b}$ (dashed line) that generate the distribution $G(z)$ for a medium that is initially entirely in the ground state. The pulse shapes are described in Eq. (3.2) with $T_{b}=100, B=12$ and $\mu=1$. 


$$
\begin{aligned}
& \Omega_{a}(z=0, \tau)=B G\left(z=\frac{B^{2}}{2 \mu}\left(T_{b}-\tau\right)\right), \\
& \Omega_{b}(z=0, \tau)=B \sqrt{1-\left[G\left(z=\frac{B^{2}}{2 \mu}\left(T_{b}-\tau\right)\right)\right]^{2}}:
\end{aligned}
$$

In this case the required input pulse shape $\Omega_{a}(z=0, \tau)$ is just a mirror symmetric replica of the desired spatial excitation profile $G(z)$. In Fig. 2 we give an example of how one can convert a ground state medium into a state characterized by a three-peaked excitation function $G(z)$. In the second figure we show the temporal profiles of the corresponding two control pulses at input, which, after having propagated through the ground state medium, leave behind the medium's excitation in exactly the desired form $G(z)$. The pulse shape of the field $\Omega_{b}(z=0, \tau)$ at early times before the second field $\Omega_{a}(z=0, \tau)$ is turned on does not influence the final excitation function. The relative abrupt turn-off at $\tau=T_{b}$ is required by our condition $\Omega(z=0, \tau)=$ const for $0<\tau<T_{b}$. This condition, however, was only imposed by us to invert relation (3.1) so as to obtain the fully aṇalytical solutions (3.2).

\section{Optical properties of media with space-dependent excitations}

Let us now assume we have a medium which has been successfully brought into the space dependent state given by $G(z)$. Let us take the three-peaked distribution shown in Fig. 2a as our working case here. How can one verify whether the medium is really precise in this state? Is there a method which allows us to retrieve the information stored in the spatially excited medium? It turns out that this can be done [19] if we inject a laser pulse with a constant amplitude field through the medium. As we will see below, a second laser field will be created inside the medium and its temporal profile at output reflects the original medium's quantum mechanical state as described by $G(z)$.

We denote the Rabi frequency of the input field by $R(\tau)$ and refer to it as the "recall" field. Its frequency is assumed to be close to the 2-3 transition frequency of the medium. A pulse of this type would have been called $\Omega_{b}$ in the previous section. Again the polarization-electric field relations of Eq. (1.3) are the key to understanding how this recall can happen. If we insert the relation $\rho_{33}=\left|\Omega_{a}(z, \tau) / \Omega(\tau)\right|^{2}$ into the nonlinear wave equation, we obtain the temporal and spatial evolution of $\rho_{33}(z, \tau)$

$$
\frac{\partial}{\partial z} \rho_{33}(z, \tau)=-\left(2 \mu / \Omega^{2}\right) \frac{\partial}{\partial \tau} \rho_{33}(z, \tau) .
$$

Here it is important to note that $\Omega(\tau)$ has been replaced with $R(\tau)$, as in this case there is only one field injected into the medium. In other words, the space-time evolution of $\rho_{33}(z, \tau)$ is entirely determined by $R(\tau)$ and $\rho_{33}(z, \tau=0)=$ $|G(z)|^{2}$. Under these boundary and initial conditions the solution for $\rho_{33}(z, \tau)$ is straightforward $\rho_{33}(z, \tau)=|G(z-X(\tau))|^{2}$. As $R(\tau)$ is turned on after time $\tau=0$, we have assumed $X(0)=0$. Returning to the trapped state relation $\rho_{33}=\left|\Omega_{a} / R\right|^{2}$, we can solve this relation for $\Omega_{a}$ which we call the signal field $S(z, \tau) \equiv \Omega_{a}(z, \tau)$. We obtain the following solution for the signal field: $S(z, t)=-G(z-X(\tau)) R(\tau)$. 
During those times for which $R(\tau)$ is constant for $0<\tau<T_{R}, R(\tau)=R$, the integral $X(\tau)$ can be simplified to $X(\tau)=R^{2}\left[\tau-\left(\tau-T_{R}\right) \vartheta\left(\tau-T_{R}\right)\right] / 2 \mu$. In other words our solution simplifies for this time interval and we obtain

$$
S(z, \tau)=-G\left(z-R^{2} \tau / 2 \mu\right) R .
$$

This shows that a second field has been created inside the medium whose temporal envelope reflects the spatial distribution of the medium $G(z)$. This, of course, opens the possibility to control laser pulse shapes using spatially prepared dielectric media.

In Fig. 3 we have injected a recall field into the medium prepared according to the three-peaked distribution $G(z)$. We show the recall and signal field as a function of $z$ and $\tau$. At the three positions where the function $G(z)$ is peaked, the signal field $S(z, \tau)$ is generated. After a characteristic propagation distance when the information transfer is complete, the signal carries all the information of $G(z)$ and travels shape invariant. Figure $3 \mathrm{~b}$ displays the recall field. The three "holes" in $R(z, \tau)$ indicate that the recall field looses some of its energy to form the signal field. After both fields have left the medium, the final state is the ground state for each atom and the memory of the original excitation function $G(z)$ is lost.

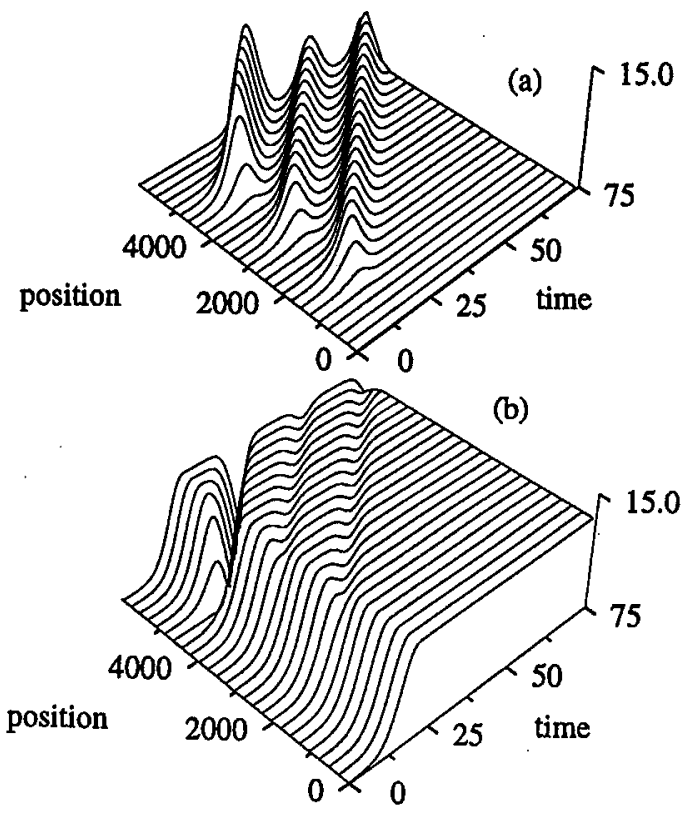

Fig. 3. Recall of spatially dependent excitations. (a) Temporal and spatial profile of the signal field $S(z, \tau)$ that is generated by the three-peaked spatial distribution $G(z)$ of Fig. 2a. (b) Temporal and spatial evolution of the recall field $R(z, \tau)$. The parameters were: $\mu=1$ and $R(z=0, \tau)=R \exp \left(-0.5\left[\left(\tau-4 \tau_{\mathrm{p}}\right) / \tau_{\mathrm{p}}\right]^{2}\right)$ for $\tau<4 \tau_{\mathrm{p}} ; \Omega_{b}(z=0, \tau)=R$ for $4<\tau / \tau_{\mathrm{p}}, R=12, \tau_{\mathrm{p}}=5$. 


\section{Discussion and conclusion}

In this review we have given an example of adiabatic control theory for a medium consisting of three-level lambda systems and driven by a pair of resonant laser pulses. This research area is just in its cradle, and many important questions need to be addressed in the future. A direct relation between the polarization and the electric field vector is the key to investigating this regime fully analytically. This direct relation is completely different to the standard perturbative expansion of nonlinear optics.

We have restricted our discussion to a very simplified model of a three-level atom. An important question of course concerns the range of applicability of the adiabaticity relation and the corresponding validity of the trapped state assumption. The validity of the adiabaticity relation (see, e.g. Ref. [6]) requires basically that the (dimensionless) product $\Omega \tau \gg 1$. This condition can be quite easily satisfied for optical transitions in dielectric media if the lasers have intensities in the range of a few $\mathrm{MW} / \mathrm{cm}^{2}$ and have pulse durations in the nanosecond range. This is also confirmed by the experimental observation of adiabatons in the study by Kasapi et al. [10]. In a previous work [9] we compared the predictions of the approximate analytical solutions based on adiabaticity with those obtained from direct numerical simulations of the fully-coupled Maxwell-Schrödinger equation and we found that the agreement is excellent.

Another equally important question concerns the applicability of the theory to dissipative systems. The trapped state condition relies on the coherence between the two lowest levels 1 and 3 . Any dissipative influence that destroys this coherence changes the dynamics sufficiently. On the other hand, any relaxation process which is associated with the upper level 2 has only a small influence on the dynamics if the Rabi frequencies are chosen larger than the relaxation rates. This is due to the fact that the population in the upper state is very small during the entire evolution, and the exchange of population happens to be restricted to levels 1 and 3 .

We finish this report with a comment about possible applications. The nearly loss-free propagation properties of adiabatons could be exploited in the long-distance transmission of optical signals. Because of the fact that the temporal features of one of the input pulses $\left(\Omega_{a}\right)$ are imprinted on the other pulse duration, the formation of adiabatons could also be advantageous in a signal replicator.

The function $G(z)$ determines obviously the optical properties for a weak optical probe field. The optical index of refraction can be directly proportional to the degree of excitation as described by $G(z)$. We have shown above that practically arbitrary excitation functions can be generated by an appropriate choice of input fields. In other words, the index of refraction in its spatial dependence can be controlled. The state 3 is typically metastable and its lifetime determines how long a medium can maintain the spatially dependent index of refraction until it relaxes back into its ground state and its normal space-independent index. The faster time scale on which the two-photon coherence decays due to energy conserving collisions, e.g., is not expected to affect the index. The combination of the recall and excitation mechanism could be exploited in the intermediate storage of optical data. 


\section{Acknowledgment}

We would like to acknowledge our collaborators J.H. Eberly, F.T. Hioe and A. Rahman in Rochester, and B.K. Clark, J.R. Csesznegi and Q. Su in Normal. We acknowledge the receipt of a travel grant under NSF grant INT 9722745 to participate at the wonderful Quantum Optics IV conference in Jaszowiec, Poland. This work has been supported by the NSF under grant \#PHY-9631245. This research was also supported in parts by a Cottrell College Science Award from Research Corporation and by Illinois State University.

\section{References}

[1] K.-J. Boller, A. Imamoglu, S.E. Harris, Phys. Rev. Lett. 66, 2593 (1991); J.E. Field, K.H. Hahn, S.E. Harris, Phys. Rev. Lett. 67, 3062 (1991).

[2] For a review on electromagnetically induced transparency, see S.E. Harris, Phys. Today 50, No. 7, 36 (July 1997).

[3] For a review, see O. Kocharovskaya, Phys. Rep. 219, 175 (1992) and articles in the special issue of Quantym Opt. 6, No. 4 (1994). For some early work on LWI, see, e.g, M.O. Scully, S.Y. Zhu, Phys. Rev. Lett. 62, 2812 (1989); S.E. Harris, Phys. Rev. Lett. 66, 1033 (1989); O. Kocharovskaya, P. Mandel, Phys. Rev. A 42, 523 (1989); A. Imamoglu, J.E. Field, S.E. Harris, Phys. Rev. Lett. 66, 1154 (1991).

[4] G. Alzetta, A. Gozzini, L. Moi, G. Orriols, Nuovo Cimento B 36, 5 (1976).

[5] R.M. Whitley, C.R. Stroud, Jr., Phys. Rev A 14, 1498 (1976).

[6] J.R. Kuklinski, U. Gaubatz, F.T. Hioe, K. Bergmann, Phys. Rev. A 40, R6749 (1989).

[7] M. Fleischhauer, A. Manka, Phys. Rev. A 54, 794 (1996).

[8] K.M. Paul, J.R. Csesznegi, R. Grobe, Laser Phys. 7, 884 (1997).

[9] R. Grobe, F.T. Hioe, J.H. Eberly, Phys. Rev. Lett. 73, 3183 (1994).

[10] A. Kasapi, M. Jain, G.Y. Yin, S.E. Harris, Phys. Rev. Lett. 74, 2447 (1995).

[11] F.T. Hioe, R. Grobe, Phys. Rev. Lett. 73, 2559 (1994).

[12] J.H. Eberly, Quantum Semiclas. Opt. 7, 373 (1995).

[13] R. Grobe, J.H. Eberly, Laser Phys. 5, 542 (1995).

[14] M.O. Scully, Phys. Rev. Lett. 55, 2802 (1985) and Phys. Rev. Lett. 67, 1855 (1991).

[15] For a review on phaseonium, see, e.g., M.O. Scully, Phys. Rep. 219, 191 (1992) and Quantum Opt. 6, 201 (1994).

[16] J.H. Eberly, A. Rahman, R. Grobe, Phys. Rev. Lett. 76, 3687 (1996).

[17] G. Vemuri, K.V. Vasada, G.S. Agarwal, Phys. Rev. A 54, 3394 (1996).

[18] J.H. Eberly, A. Rahman, R. Grobe, Laser Phys. 6, 69 (1996).

[19] J.R. Csesznegi, R. Grobe, Phys. Rev. Lett. 79, 3162 (1997).

[20] J.R. Csesznegi, B.K. Clark, R. Grobe, Phys. Rev. A, to be published. 